ACTA UIVERSITATIS CIBINIENSIS - TECHNICAL SERIES

Vol. LXIV No 12014

\title{
GENERALIZED MODELING OF THE HUMAN LOWER LIMB ASSEMBLY
}

\author{
IOANA COFARU \\ University „Lucian Blaga” Sibiu, Romania, oioanalogistictop@yahoo.com \\ IULIA HUZU \\ University „,Lucian Blaga”Sibiu, Romania, huzuiulia@yahoo.com
}

\begin{abstract}
The main reason for creating a generalized assembly of the main bones of the lower human member is to create the premises of realizing a biomechanic assisted study which could be used for the study of the high range of varieties of pathologies that exist at this level. Starting from 3D CAD models of the main bones of the lower human member, which were realized in previous researches, in this study a generalized assembly system was developed, system in which are highlighted both the situation of an healthy subject and the situation of the situation of a subject affected by axial deviations. In order to achieve these purpose reference systems were created, systems that are in accordance with the mechanical axes and the anatomic axes of the lower member, which were later generally assembled in a manner that provides an easy customization option.
\end{abstract}

Keywords: assembly, modeling, reconstruction.

\section{Introduction}

Computer assisted modeling finds its full utility in the biomedical field. As the study has as objects real human parts that cannot be studied as real experimental objects, the simulation and the modeling methods prove their full effectiveness in this field, a field in which model based study is the most indicated.

The possibility of a generalized modeling possibility of the lower human member is of a great importance given the wide range of pathological situations that can occur.

For example, if we would take into consideration only the derivations in the frontal plane of the foot(all perfectly realizable with a generalized assembly), we have the possibility to simulate, evaluate and verify just knee pathological situations such as genu varum, genu valgum, sprains, osteoarthritis, bone setting the responsible bones for misalignment, namely tibia, femur or both, establishing treatment strategies depending on the angles, elements of great importance both in terms of preoperative and teaching perspectives, possible deformations and tension checking on a situation resulting in custom assembly, being able to determine what has occurred and how we can intervene on the geometric values.

Taking into consideration that for the knee articulation there are two more planes (sagital and tranverse) with as many malformations and usages as in the other planes and that the way in which the assembly is made allows all of these approaches also for the other articulations of the leg (ankle and hip), we can observe the complexity of such system and the necessity to realize a generalized assembly system. contribution.

The realization of such a generalized assembly system can be considered as an original important 
2. Reference systems. Trihedral. Planes. Axis. Origins.

Figure 2: Reference system

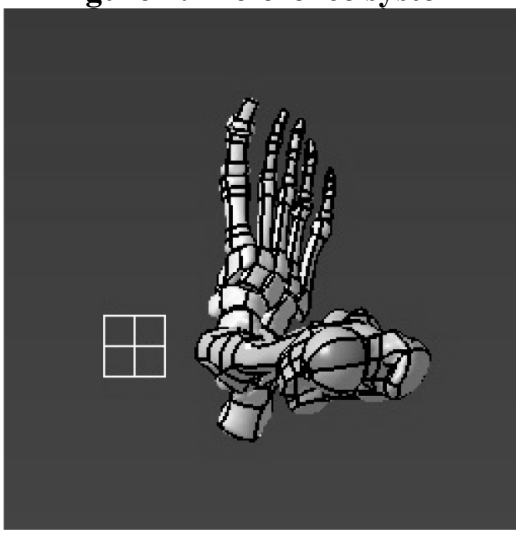

Figure 3: Inferior origin of the tibia $\mathrm{OT}_{\mathrm{T} 1}$

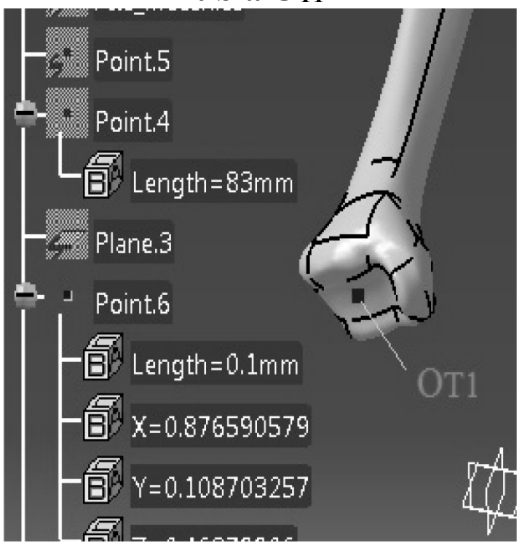

Figure 4 : Superior origin of the tibia $\mathbf{O}_{\mathrm{T} 2}$

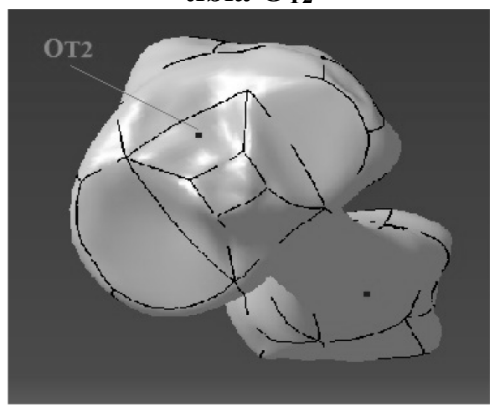

As any assembly starts, it is necessary to create reference systems on which we can relate the components that will be assembled. To obtain a real general character, it is important to use as references, systems of the human body, commonly used in medicine. We will use reference planes of the human body (front, sagittal, transverse), its axis (mechanical and anatomical) angular sizes established for a healthy subject and also different situations that are characteristic for pathological deviations. In this way, the assembly will take into account all these geometric references. The first step was to choose a principal reference system, on which all the lower limb bones were assembled. This reference system is triortogonal and has the origin placed on the ground projection of the center of gravity of the human body.

To locate the exact reference system, in Figures 1 and 2 the whole leg is presented as an orthogonal projection in the sagittal plane, and the top or cross ones. Planes of the reference system are: $\mathrm{XOZ}$ which is the plan that actually sits the foot, YOZ is the symmetry plane that divides the body into two symmetrical parts (left and right) plan very important in achieving balancing and mirroring techniques in, for comparison and possible reconstruction of the affected areas. And finally XOY plane perpendicular to the first two and passing through the center of gravity of the body. Models tibia, femur and foot that have been calculated in relation to the system described above. Since the subject of this paper concerns on the knee joint, reference elements that make up the joint - femur or tibia- will be created. Below are some important points that will determine the origins for the reference systems and points that will deter-mine the limb axis. Figure 3 presents the origin of the lower tibia OT1 located in the center of symmetry of the contact area, between the tibia and foot dome. OT 2 upper

\section{Figure 1: Reference system}

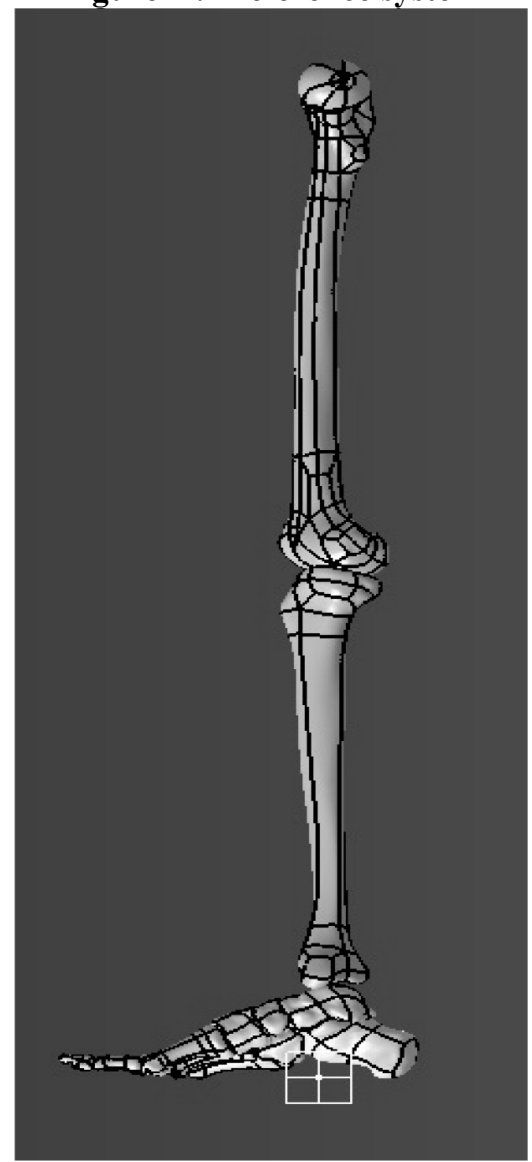

Figure 5 : Modelling the axis of the tibia

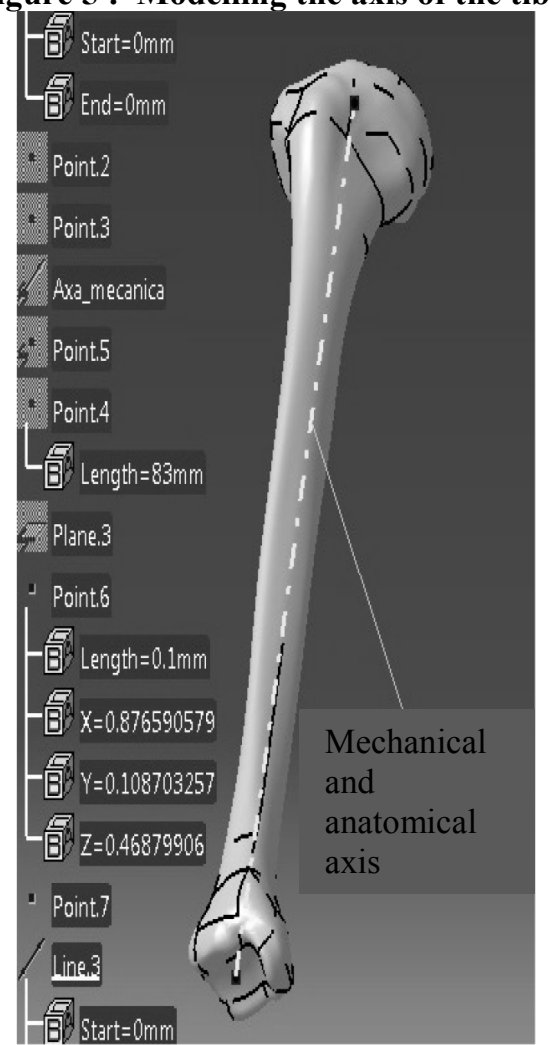


Figure 6 : Important points of the femur

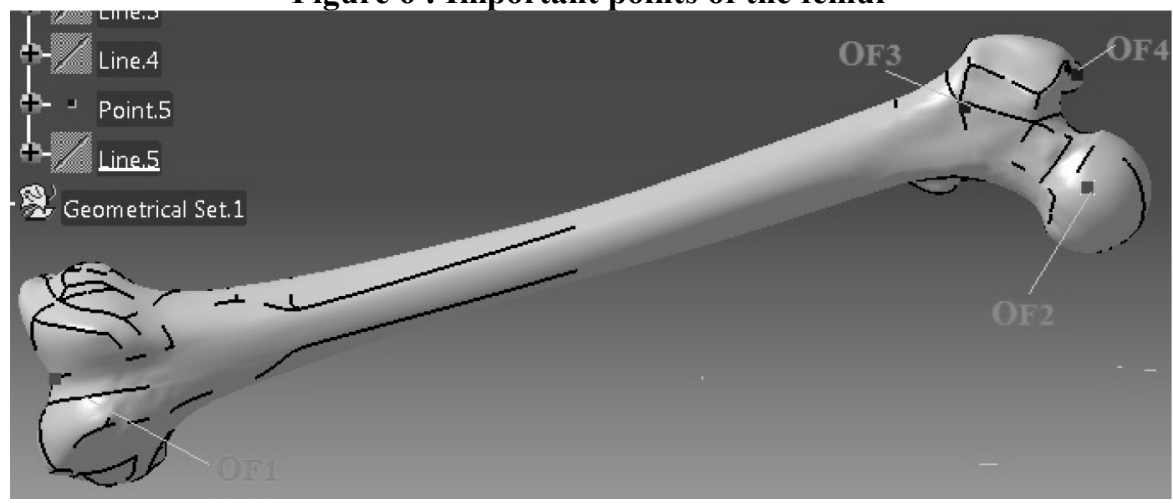

Figure 7 : The main axis of the femur

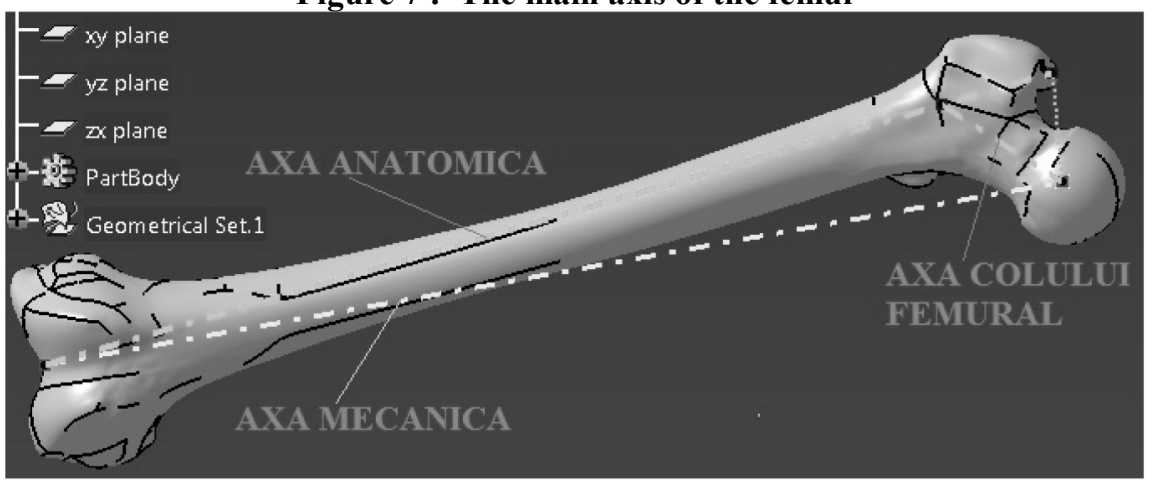

tibia origin, located at the center of symmetry of the tibia spleen (Figure 4) .Using the two origins created the model, we can insert the mechanical axis of the tibia joining the knee joint center to the center of the ankle joint (Figure 5).In the case of the tibia, the mechanical axis is the same as the anatomical axis of symmetry of the bone. In the case of the femur, the more complex configuration and positioning in the human frame requires the insertion of multiple origins and axes (Figure 6.7) .A first home OF1 is inserted between the two femoral condyles, in the center of symmetry of the knee. The second important point is introduced OF2 which is center of the femoral head, which can be assimilated to a sphere, from a geometric point of view. Point OF3 was inserted at the junction anatomical axis of the femur and femoral neck axis, and OF4 is inserted into the tip of the great trocanter.

The axis of the femur can be placed on the model, by setting them as follows:

1) mechanical axis of the femur joining points OF1 OF2

2) anatomical axis is determined by points OF1 and OF4

3) femoral axis between points $O F 3$ and $O F 2$

Axis represented by the dotted line joins the points OF2 and OF4 and generally, on the healthy individual, is perpendicular to the mechanical axis. It presents more importance from the point of view of the relative positioning, than of the analysis of pathologic conditions.

The introduction of these axes model is important because it highlights also other geometric elements, such as angles or head positioning to a healthy or an ill subject.

\section{General assembly. Skeleton systems. Creating links with pathological cases of lower limb}

Making the assembly of the bones as they were previously modeled, will be made according to the methodology which is available in Catia V5R20, consisting in that that it calls the existing components (Existing Components) in an Assembly Design window which afterwards will be constrained. For an accurate assembly, we must take into account that the contact surfaces are changing, depending on the subject studied. For example, in the knee joint contact areas will be different when we will compare that of a healthy individual to another that is suffering of gonarthrosis. Our aim is to create a system that allows general assembly in which we can study the biomechanical of both: a healthy leg and of one affected by various diseases.

For this purpose, we used Axis Systems function in Catia V5 and in origin points from the preceding paragraph, triortogonale axis systems were created (Figures 8-10). The triortogonal axis systems were called Skeleton femur, tibia Skeleton and Skeleton leg. The skeletons are actually reference systems that remain fixed, any changes will be reported in relation to the reference systems. These axis systems that were created in this way, taking into account the assembly, the normal anatomical contacts 
and also the constraints imposed on them, can simulate various pathological situations. The main idea is that the systems were chosen like triortogonal axis systems Catia V5, as Euler systems, in order to execute relative rotation between their planes. Geometric elements taken into account for the assembly were:

1) coincidence of $Y$ axis of the skeletons with mechanical axes for each bone, planes $\mathrm{YOZ}$ of the skeleton are parallel the frontal plane of the human body

2) XOZ planes are horizontal planes corresponding with the contact areas of the knee and ankle, XOY planes are parallel to the sagittal plane of the body.

The constraints applied for the realization of the set are:

1) constraint fixing the axis system Skeleton

2) coincidence of mechanical and Y axes of "Skeleton "

3) coincidence of planes and planes XOZ contact appropriate

4) parallelism between the planes of the human body and the corresponding planes "Skeleton"

In Figure 12 the assembled leg bones are shown in orthostatic position and in Figure 11 systems Skeleton from the ankle and knee are inserted. All the system axes are able to perform movements of rotation and translation between the corresponding axes resulting relative positions that may characterize different pathological situations.

Figure 12 : Lower limb bone assemby

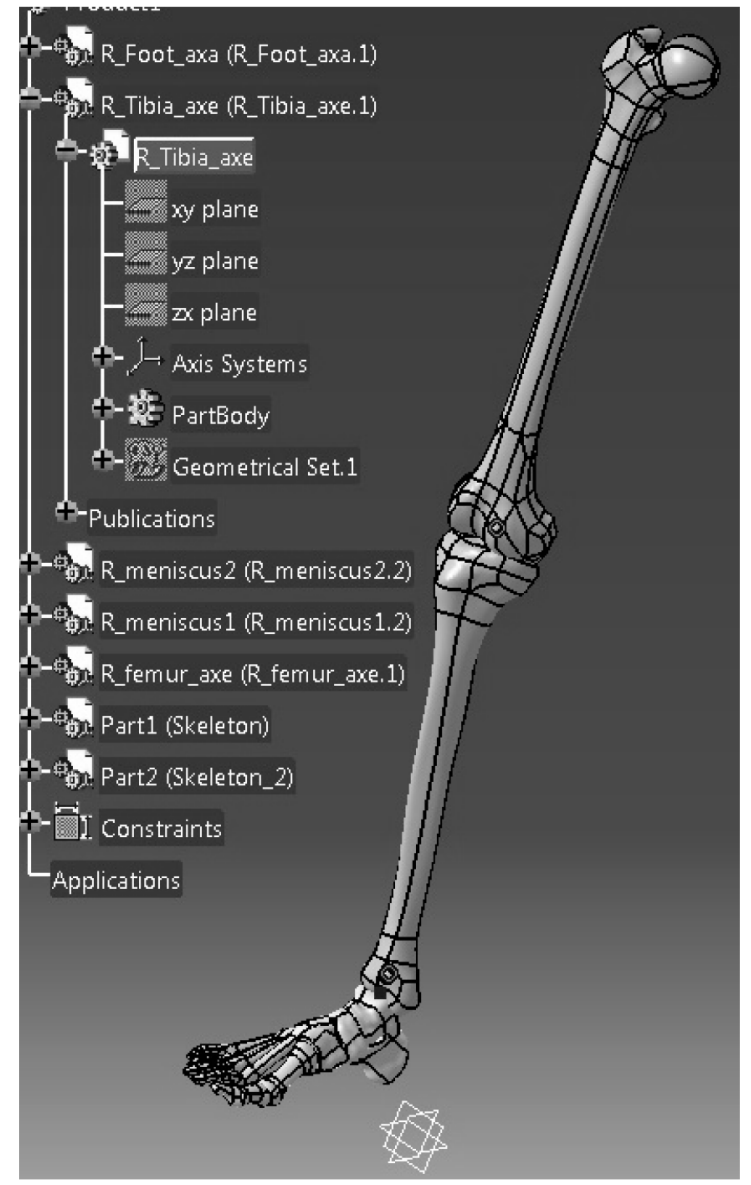

Figure 8 : Femur Skeleton

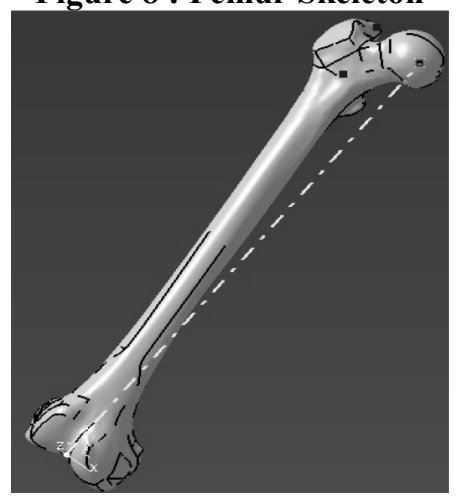

Figure 9 : Foot Skeleton

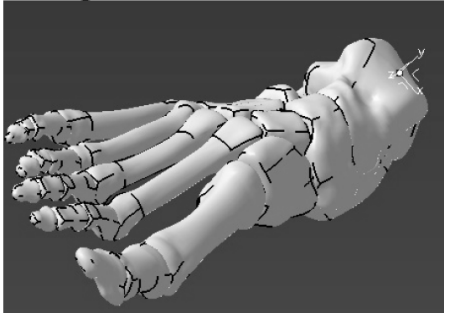

Figure 10 : Tibia Skeleton

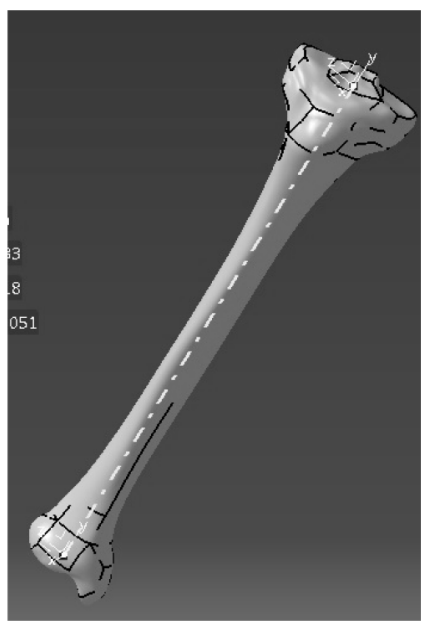

Figure 11 : Skeleton systems assembled

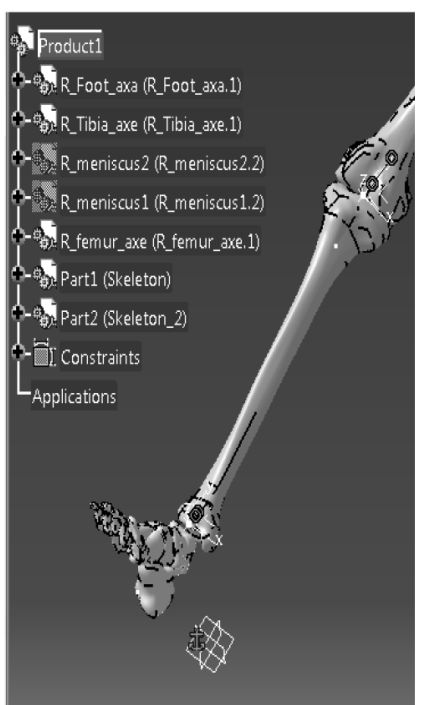


Figure 13 : Varus equinus

\begin{tabular}{|c|c|}
\hline Axis System Definition & $? \underline{x}$ \\
\hline Axós system type: Euler angles & \pm \\
\hline Origin: Point.2 & \\
\hline Angle 1: Odeg & 图 \\
\hline Angle 2: Odegl & 图 \\
\hline Angle 3: 30deg & 眯 \\
\hline$\square$ Current Righthanded & More... \\
\hline O OK & 9 Cancel \\
\hline
\end{tabular}

Figure 15 : Genu Varum deformation
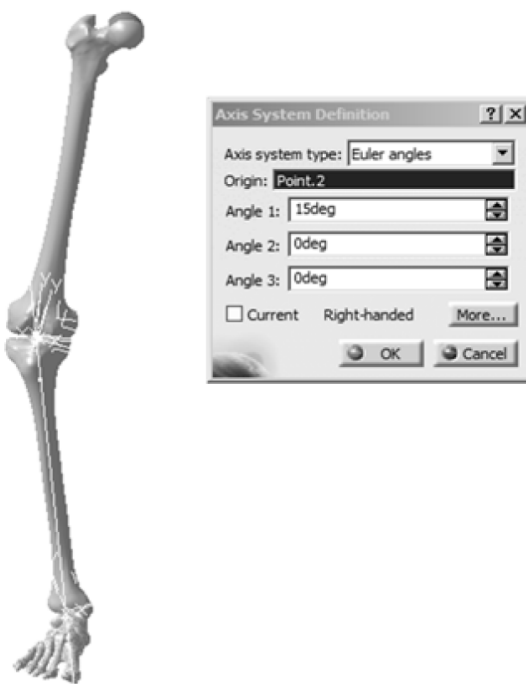

Figure 17 : Genu Flexum deformation

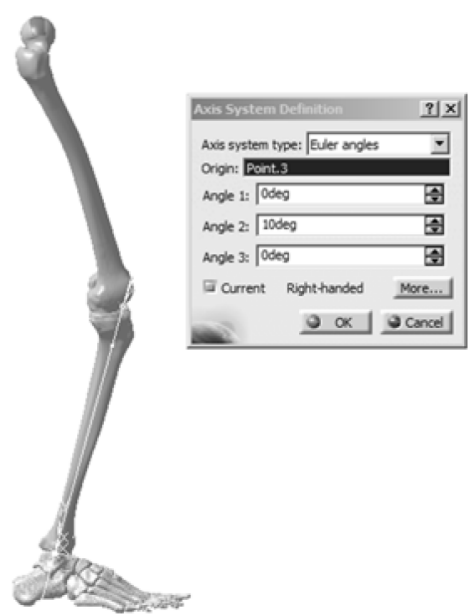

Figure 14 : Knee sprain

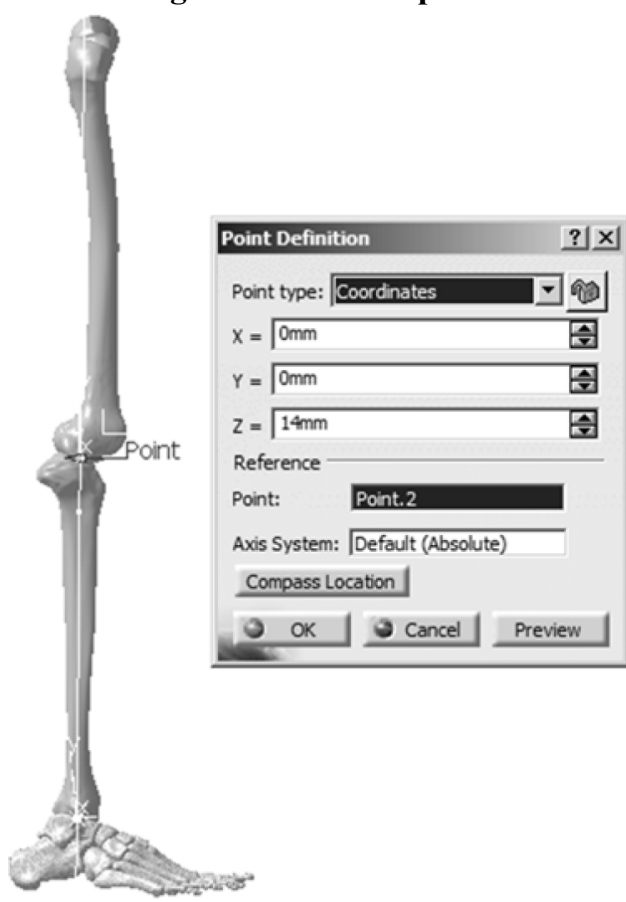

Figure 16 : Genu Valgus deformation

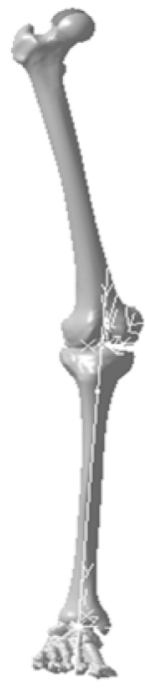

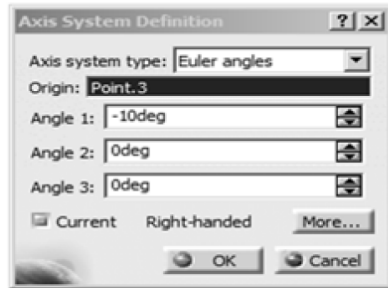

Figure 18 : Genu Recurvatum deformation

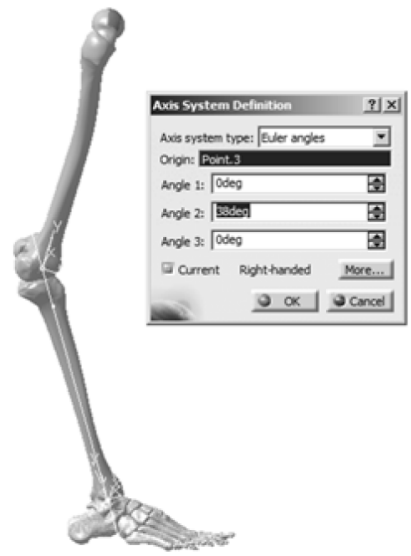




\section{Conclusions}

3D assembly models in generalized manner of the lower limb bones, offer the possibility of a biomechanical study of both a healthy lower member and of a member affected by different diseases. The research is of a great importance from both an academic perspective and from a pre surgery point of view. The focus was on the axial deviation of the foot (rotations, translations, generalized ensemble can be customized in many pathological situations (genu varus, genu varum, genu recurvatum, genu flexum, varus equinus, etc). (Figure 13-18)

\section{References}

- Cofaru I..I., Brîndaşu P.D., Cofaru N.F. and Roman L. (2013), Computer Simulation Paradigm Regarding the Structure and Mechanical Characteristics of Human Long Bones, ISCS, Timişoara.

- Dan_Ek J. (2007), Numerical Analysis Of The Total Knee Joint Replacement - Modelling In Comsol, University Of West, Bohemia.

- Huzu E.I., Cofaru I.I., Cofaru N.F.(2013), Research regarding CAD modeling of the human lower limb bones, Annals of the Oradea University, Fascicle of Management and Technological Engineering, issue \#1.

- Yao J., Tamez-Pena J.G., Totterman S.M.S., Salo A.D., Lerner A.L. (2003), Finite Element Modeling Of In Vivo Knee Joint Contact Under Axial Loading. 